\title{
A three-phases model for the simulation of landslide-generated waves using the improved conservative level set method
}

\author{
Jia MAO ${ }^{\mathrm{a}, \mathrm{b}}$, Lanhao ZHAO ${ }^{\mathrm{a},{ }^{*}}$, Xunnan LIU $^{\mathrm{a}}$, Jing CHENG $^{\mathrm{a}}$, Eldad AVITAL ${ }^{\mathrm{b}}$ \\ ${ }^{a}$ College of Water Conservancy and Hydropower, Hohai University, Xikang Rd., Nanjing \\ 210098, PR China \\ ${ }^{b}$ School of Engineering \& Materials Science, Queen Mary University of London, Mile End \\ Rd., London E1 4NS, UK
}

\begin{abstract}
This paper introduces a three-phases model based on the finite element method to simulate the generation and propagation of landslide-generated impulse waves, and this model can be employed to predict and prevent wave-induced hazards. The fluid-like landslide mass is treated as a non-Newtonian viscoplastic fluid. The motion of landslides, water and air is modelled by the incompressible Navier-Stokes equations and the interfaces between these three phases are captured with the $n$-phases improved conservative level set method which can preserve mass and provide precious interface parameters, including normals and curvatures. The conservative feature of this method is proven by the threephases Zalesak slotted disk test case. This method is then adopted to simulate the impulse wave generated by the Lituya Bay landslide and the current outputs are compared with other existing results. Finally, this verified model is utilized to model the impulse waves generated by the Halaowo landslide near the Xiangjiaba Dam in the Jinsha River and the results could provide references for further protective activities.
\end{abstract}

KEYWORDS: impulse waves, landslide, three-phases flows, improved conservative level set, finite element method, characteristic-Galerkin procedure

* Corresponding author:

Lanhao ZHAO

Tel.: +86 13914733646

Fax: +862583731332

E-mail address: zhaolanhao@hhu.edu.cn 


\section{Introduction}

Landslides are frequent and severe geological activities which can lead to disastrous damage. Compared with landslides which slide in an open area, those impacting into a reservoir could cause more catastrophic consequences because of the generated destructive waves. In extreme situations, waves could overtop a dam and inundate vast areas along the shoreline. One of the deadliest disasters is the Vajont Reservoir landslide in 1963. The landslide mass generated a wave with a height of $250 \mathrm{~m}$ and claimed around 2000 lives. It is worth noting that there are tremendous potential landslides in reservoirs due to the frequent change of water levels. The Shanshucao landslide (about $4 \times 10^{7} \mathrm{~m}^{3}$ ), which is one of 3028 landslides observed in the Three Gorges Reservoir in China, lost stability and destroyed a local hydropower station in 2014. Considering the large number of potential landslides existing in proximity of reservoirs and the severe losses, it is of great importance to carry out research on landslide-generated waves to predict the slide path of the unstable mass, the inundation area of waves and the overtopping possibility. Based on the predictive work, corresponding preventions should be performed, including slope reinforcements, controlled blasting of parts of the slide and early warnings of possible disasters.

Most of the existing researches about landslide-generated waves can be classified into four categories, analytical equations, physical experiments, numerical simulations and empirical estimations derived from field data, physical experiments or numerical simulations. In the initial stage, analytical equations $[1,2]$ are proposed to estimate wave parameters roughly. The estimations are determined by simplified assumptions of landslides, water and topography and the results could vary over an order of magnitude by applying different hypotheses. To study the general behaviour of landslides and generated waves, laboratory experiments [3-9] in both two-dimensions (wave flume geometry) and three-dimensions (wave basin geometry) are performed. However, it consumes abundant time, labour and money when more experiments should be carried out due to the application of different parameters. Moreover, the accuracy of results could be influenced by scale effects [10] and results cannot be recorded in all points.

To lower the entire cost and study the characteristics of landslides and waves in detail, numerical simulation methods attract the attention of researchers around the world. Initially, numerical simulations of landslide-generated waves are made under different assumptions due to the complexity of this problem and the limitation of the computer capacity. Depending on the hypothetical degree adopted to describe the movement of the wave in numerical 
models, the mathematical formulations can be divided into four types, namely, shallow water equations (SWEs) [11-13], Boussinesq-type wave equations (BWEs) [14-16], potential flow equations (PFEs) [17, 18] and the Navier-Stokes equations (NSEs) [19-23]. Among these methods, the general SWEs methods are appropriate for the simulation of the wave propagation process but are weak in representing the drastic phenomena during the wave generation process since the vertical acceleration is ignored. This shortcoming can be resolved by some improved wave models, including SWASH (Simulating WAves till SHore), which considers the effect of the vertical acceleration by the added non-hydrostatic pressure gradient. BWEs and PFEs models are applied to improve the order of accuracy. However, most of these existing BWEs and PFEs models are used to simulate rigid landslides by treating the landslide mass as moving boundary and ignoring the strong coupling between different phases. Compared with the former three models, NSEs models behave better in accuracy and application range. According to the conceptualization of landslides, some of the numerical models [24-26] treat the landslide mass as a rigid body and set the slide kinematics in the mass centre. However, this assumption is idealized as almost all actual landslides would deform in the sliding stage. In most cases, many physical experiments and numerical simulations have found that the wave height would be overestimated when treating the landslide as a rigid body [27-29]. However, an opposite phenomenon can be observed when there is no smooth transition at the toe of the slope [8]. In this situation, rigid slide may stop immediately whilst a granular slide runs-out further and thus transforms more energy to the wave. It is more accurate to take the deformability into consideration, especially when the landslide mass deforms drastically.

After the milestone study carried out by Quecedo et al. [30], the three-phases model gained more and more attention since it avoids the two assumptions mentioned above, namely the neglect of the vertical acceleration and the strong coupling between different phases. NSEs were applied to model the motion of landslides, water and air, which are three phases included in the landslides penetration stage, and the Level Set (LS) method was used to capture interfaces between different phases. The main advantage of this method lies in the fact that the strong coupling between landslides, water and air is considered, while the air phase is seldom taken into consideration in previous studies. Besides, they suggested that the non-Newtonian viscoplastic fluid model, which is a fluid-like model, can be utilized to model landslides when the slide mass slides down with a high speed. Considering the high moisture content and thick alluvium deposits of slopes in reservoirs, it is appropriate to treat this kind of landslides as fluid-like. Based on the three-phases model, many researchers [21, 31] 
improved the simulation accuracy of the landslide-generated waves and extended the calculation to subsequent stages, including wave propagation and run-up. As the study objects herein are waves generated by landslides in reservoirs, the three-phases model, which treats landslides as fluids, is adopted in this paper.

When the three-phases model is employed to simulate landslide-generated waves, an accurate and efficient technique for the description of interfaces is crucial since interfaces could undergo drastic changes, including breaking, overturning and merging, caused by the strong interaction between different phases. It remains challenging to provide a conservative interface representing method, which can describe interfaces accurately. Typical methods of describing interfaces include Smoothed Particle Hydrodynamics (SPH) [32-35], Volume of Fluid (VOF) method [36-38] and the LS method [39, 40]. Even though SPH method is widely known as a numerical technique which is able to solve the partial differential equations, it can also be used to represent interfaces. SPH is able to enhance the conservative property without advection errors within the entire calculation process. However, SPH has a high demand in computational cost and the modelling of boundary conditions is challenging. Besides, SPH method is unstable when tracking interfaces with large deformations. VOF method also exhibits excellent mass conservation property, but it needs complicated interfaces reconstruction. Meanwhile, it is challenging to calculate normals of interfaces exactly with VOF method because of the discontinuity of the applied color function. Compared with these two methods mentioned above, the LS method is simply and efficient to capture the front, while mass loss happens when advecting and re-initializing the indicator function. To improve the LS method, Olsson and Kreiss [41, 42] proposed a mass conservation method, which is named as the conservative Level Set (CLS) method. Even though the mass conservation property of the advection step can be ensured, both the normals and curvatures of interfaces are sensitive to small spurious oscillations because of the intrinsic shortage of the Heaviside function. Hence, the CLS method cannot provide precise normals and curvatures of interfaces, which should be used in the re-initialization process. Combining the advantages of the LS method and the CLS method, the improved conservation Level Set (ICLS) method, which can represent interfaces in a conservative way, was proposed by Zhao et al. $[43,44]$. However, this method can only be used to capture the interface of two-phases flow until now.

In this paper, a three-phases model for the simulation of landslide-generated waves is proposed. This model applies a proper rheological model, namely the non-Newtonian viscoplastic fluid model, to describe the deformable fluid-like landslide mass and employs 
NSEs to simulate the motion of the three phases in the penetration region. To model three phases in the generation of landslide-generated waves, the previous ICLS method needs to be expanded from two-phases to $n$-phases. All the governing equations are discretized with the characteristic-Galerkin procedure. Compared with previous publications, this new model can represent interfaces more accurately by utilizing the expanded $n$-phases ICLS method, which can provide precise interface characteristics and preserve mass conservation property.

This paper is organized as follows. In Section 2, the governing equations, constitutive equations and the implementation of the ICLS method for $n$-phases flows are described. In Section 3, the numerical schemes including the characteristic-Galerkin procedure, discretization of NSEs and ICLS functions are shown in detail. In Section 4, the mass conservation property of this proposed method is verified by a benchmark test, namely the three-phases Zalesak slotted disk. Then, the proposed method is validated by comparing the current simulation outputs of the Lituya Bay landslide waves with the existing published results. Subsequently, this verified method is employed to predict impulse waves generated by the Halaowo landslide in the Xiangjiaba Reservoir. At the end of this paper, in Section 5, the conclusions are given.

\section{Mathematical model}

\subsection{Governing equations}

Considering immiscibility of the three phases interacting with each other, the viscous incompressible NSEs are employed to govern their motion:

$$
\begin{gathered}
\nabla \cdot \boldsymbol{u}=0 \\
\frac{\partial \boldsymbol{u}}{\partial t}+\nabla \cdot(\boldsymbol{u} \otimes \boldsymbol{u})=\frac{1}{\rho} \nabla \cdot \tau-\frac{1}{\rho} \nabla p+\boldsymbol{f}_{b}
\end{gathered}
$$

where $\nabla$ represents the gradient operator, $u$ indicates the velocity vector, $t$ is time, $\tau$ is the viscous stress tensor which is given in Section 2.2, $p$ represents the pressure and $f_{b}$ indicates the body force. Other forces not mentioned are not involved in the simulations in this paper. The material properties, such as density $\rho$, are determined in Section 2.3.

\subsection{Constitutive equations}

The whole mass can be modelled with one constitutive equation when the landslide mass slides down with a high speed [30]. Based on this simplification, Chen and Ling [45] presented a model for fluid with the viscoplastic property. The constitutive equation for the fluid-like slide is 


$$
\boldsymbol{\tau}=\left(\frac{\tau_{y 0} \cos \phi+p \sin \phi}{\sqrt{I_{2 D}}}+2 \mu_{1}\left(I_{2 D}\right)^{\frac{\eta_{1}-1}{2}}\right) \boldsymbol{D}+\frac{4}{3} \mu_{2}\left(4 I_{2 D}\right)^{\frac{\eta_{2}-1}{2}} \boldsymbol{D}^{2}
$$

in which $I_{2 D}$ is the second invariant of $\boldsymbol{D}, \tau_{y 0}$ is the fluid yield stress, $\phi$ is the internal friction angle, $\mu_{1}$ and $\mu_{2}$ are coefficients related to viscosity and $\eta_{1}$ and $\eta_{2}$ are exponents associated with the flow behavior.

A further simplification is carried out by Quecedo et al. [30] by removing the second term in Eq. (3):

$$
\boldsymbol{\tau}=\left(\frac{\tau_{y 0} \cos \phi+p \sin \phi}{\sqrt{I_{2 D}}}+2 \mu\left(I_{2 D}\right)^{\frac{\eta-1}{2}}\right) \boldsymbol{D}
$$

Different rheological models, such as Newtonian, Bingham and Frictional fluid model, can be obtained as particular cases of Eq. (4) and their parameters are shown in Table 1.

\begin{tabular}{lccc}
\hline Rheological model & $\tau_{y 0}$ & $\phi$ & $\eta$ \\
\hline Newtonian fluid & 0 & 0 & 1 \\
Bingham fluid & arbitrary value & arbitrary value & 1 \\
Frictional fluid & 0 & arbitrary value & 1 \\
\hline
\end{tabular}

Table 1 Parameters for different rheological models.

\subsection{Improved conservative Level set method}

Both the LS method and the CLS method have drawbacks, non-conservation of mass and incorrect surface normal direction respectively. To overcome these deficiencies, the ICLS method is given by combining their merits together.

To represent the interface in the CLS method, a hyperbolic tangent function is employed

$$
H(\psi)=\frac{1}{2}(1+\tanh (\psi / 2 \varepsilon))
$$

in which $\psi$ is the signed distance function in the LS method and $\varepsilon$ is a spreading width of $H$.

For incompressible flow, the CLS function can be described as

$$
\frac{\partial H}{\partial t}+\nabla \cdot(\boldsymbol{u} H)=0
$$

where $u$ stands for the fluid velocity field.

A distortion of $H$ can be observed when $H$ is adverted because of numerical errors and velocity changes. To avoid this problem, a re-initialization step of $H$ is needed 


$$
\frac{\partial H}{\partial \tau}+\nabla \cdot(H(1-H) \boldsymbol{n})=\nabla \cdot(\varepsilon(\nabla H \cdot \boldsymbol{n}) \boldsymbol{n})
$$

where $n$ presents the normal vector before doing re-initialization.

In the CLS method, $\boldsymbol{n}$ is given as

$$
\boldsymbol{n}=\frac{\nabla H}{|\nabla H|}
$$

However, it is improper to use Eq. (8) to obtain the normal vector away from the interface, i.e. where $H$ is close to 0 or 1 . This can be verified by Eq. (5) and the property of signed distance function, $|\nabla \psi|=1$. The gradient modulus of $H$ can be written as

$$
|\nabla H|=\frac{1-(2 H-1)^{2}}{4 \varepsilon}
$$

The value of $|\nabla H|$ is near 0 when the point is away from the interface, which will lead to the normal vector points to an arbitrary direction according to Eq. (8). To prevent this problem, the calculation of normal vector for points at a distance from the interface is given in the ICLS method.

$$
\boldsymbol{n}= \begin{cases}\nabla \psi /|\nabla \psi| & \text { if }|\psi|>\delta \\ \nabla H /|\nabla H| & \text { if }|\psi| \leq \delta\end{cases}
$$

where $\delta$ is a certain threshold level.

It is important to mention that $\psi$ should be calculated (Eq. (11)) and re-initialized (Eq. (12)) before being used to calculate the normal vector. A re-initialization method proposed by Sussmana [46] is adopted and the equations usually undergo several steps of iteration.

$$
\begin{gathered}
\frac{\partial \psi}{\partial t}+\nabla \cdot(\boldsymbol{u} \psi)=0 \\
\frac{\partial \psi}{\partial \tau}+\nabla \cdot(\tilde{\boldsymbol{u}} \psi)=S\left(\psi^{n}\right)+\psi \nabla \cdot \tilde{\boldsymbol{u}}
\end{gathered}
$$

in which $\tilde{\boldsymbol{u}}=S\left(\psi^{n}\right) \frac{\nabla \psi}{|\nabla \psi|}, S()$ is the sign function and $\tau$ is a fictitious time step.

In the ICLS method, the update of $\psi$ is limited to the points which are away from the interface and it can guarantee the right interface position. $\psi$ for the points near the interface is calculated by the reverse of Eq. (5)

$$
\psi=2 \varepsilon \tanh ^{-1}(2 H-1)
$$


After getting the correct normal directions, the re-initialization can be done according to Eq. (7), which is able to reserve the conservation property. Both the correctness of the interface position and the conservation of mass can be guaranteed by using the ICLS method.

To give the values of the material properties including density and viscosity for $n$-phases flow, the indicator matrices $A_{n \times(n-1)}$ and $B_{n \times 1}$ can be used to identify the domains occupied by different fluids. $a_{i j}(i=1, n ; j=1, n-1)$ and $b_{k 1}(k=1, n)$ are the matrix elements. As indicated in Fig. 1, the interfaces between $\Omega_{1}, \Omega_{2}, \Omega_{3}$ are captured by $A_{3 \times 2}$ and $B_{3 \times 1} . \Gamma_{12}$ represents the interface between sub-domains $\Omega_{1}$ and $\Omega_{2}$. The meaning of $\Gamma_{23}$ and $\Gamma_{31}$ is similar as for $\Gamma_{12}$. Then, the material property of each domain $M P_{i}(i=1,2,3)$ can be calculated as:

$$
M P_{i}=M P_{1}+b_{i 1}
$$

in which $B_{3 \times 1}=A_{3 \times 2} \cdot\left(\begin{array}{l}M P_{2}-M P_{1} \\ M P_{3}-M P_{2}\end{array}\right), A_{3 \times 2}=\left(\begin{array}{cc}0 & 0 \\ 1 & 0 \\ 1 & 1\end{array}\right)$.

More generally, indicator matrices $A_{n \times(n-1)}$ and $B_{n \times 1}$ are defined as follows to expand this definition to arbitrary phases

$$
A_{n \times(n-1)}=\left(\begin{array}{cccc}
0 & 0 & \cdots & 0 \\
1 & \ddots & a_{j i} & 0 \\
1 & \ddots & \ddots & \vdots \\
\vdots & a_{i j} & \ddots & 0 \\
1 & 1 & \cdots & 1
\end{array}\right), B_{n \times 1}=A_{n \times(n-1)} \cdot\left(\begin{array}{c}
M P_{2}-M P_{1} \\
M P_{3}-M P_{2} \\
\vdots \\
M P_{n}-M P_{n-1}
\end{array}\right)
$$

in which $a_{i j}=\left\{\begin{array}{ll}1 & i>j \\ 0 & i \leq j\end{array}\right.$.

The material properties $M P_{i}(i=1,2, \ldots, n)$ can be calculated with Eq. (14).

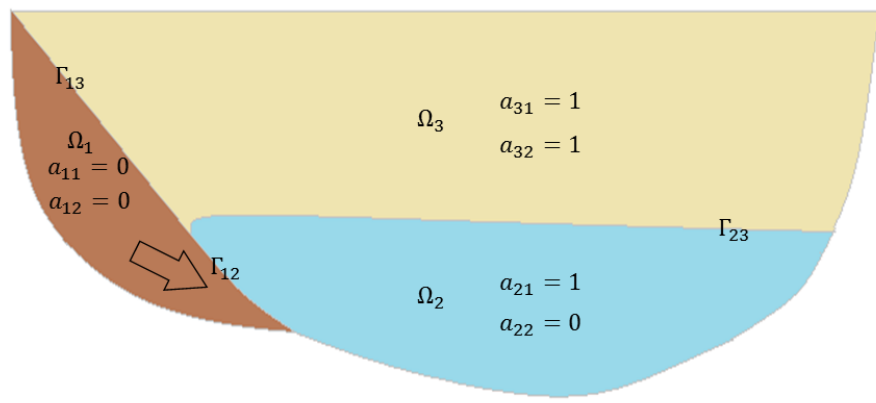

Fig. 1 Description of a three-phases flows problem using indicator matrices. 


\section{Numerical model}

\subsection{Characteristic-Galerkin procedure}

In this section, the characteristic-Galerkin procedure [47] is described in detail. Based on this method, the discretized expression of a general conservative equation is obtained.

A general conservative equation can be written as

$$
\frac{\partial \Phi}{\partial t}+\nabla \cdot \boldsymbol{F}+\nabla \cdot \boldsymbol{G}+Q=0
$$

where $\Phi$ is an unknown scalar or vector, $\boldsymbol{F}$ represents the convective flux, $\boldsymbol{G}$ indicates the diffusion flux and $Q$ is the source term.

Removing the third-order terms, the time discretization of the above equation along the characteristic gives

$$
\Delta \Phi=-\Delta t(\nabla \cdot \boldsymbol{F}+\nabla \cdot \boldsymbol{G}+Q)^{n}+\frac{\Delta t^{2}}{2} \boldsymbol{u} \cdot \nabla(\nabla \cdot \boldsymbol{F}+Q)^{n}
$$

The unknown variable can be discretized with the standard Galerkin procedure

$$
\Delta \Phi=N \Delta \bar{\Phi}
$$

where $\bar{\Phi}$ indicates the values on nodes and $N$ stands for the weighting functions for the field $\Phi$.

The incremental quantity of the unknown variable on nodes can be calculated by making the weighting functions equal to the shape functions

$$
\begin{aligned}
\int \boldsymbol{N}^{T} \boldsymbol{N} d \Omega \Delta \bar{\Phi}= & -\Delta t\left[\int \boldsymbol{N}^{T}(\nabla \cdot \boldsymbol{F}+Q) d \Omega-\int \nabla \boldsymbol{N}^{T} \boldsymbol{G} d \Omega\right] \\
& -\frac{\Delta t^{2}}{2} \int \nabla\left(\boldsymbol{N}^{T} \boldsymbol{u}\right)(\nabla \cdot \boldsymbol{F}+Q) \cdot d \Omega-\Delta t \int \boldsymbol{N}^{T} \boldsymbol{G} \cdot \boldsymbol{n} d \Gamma
\end{aligned}
$$

\subsection{Discretization of Navier-Stokes equation}

The fractional step algorithms given by Chorin [48] are widely used in discretizing the NSEs because of its accuracy and efficiency. The detailed information about the implementation of this method is described in the following. To make the proposed method in this paper applicable in dealing with both explicit and implicit problems, the governing equations are rewritten by employing two parameters namely $\theta_{1}, \theta_{2}$

$$
\begin{gathered}
\nabla \cdot \boldsymbol{u}^{n+\theta_{1}}=0 \\
\frac{\Delta \boldsymbol{u}}{\Delta t}+\nabla \cdot(\boldsymbol{u} \otimes \boldsymbol{u})=\frac{1}{\rho} \nabla \cdot \boldsymbol{\tau}-\frac{1}{\rho} \nabla p^{n+\theta_{2}}+\boldsymbol{f}_{b}
\end{gathered}
$$


where the parameters $\theta_{1}$ and $\theta_{2}$ can be chosen in the range [0,1]. For $0.5 \leq \theta_{1} \leq 1$ and $0.5 \leq \theta_{2} \leq 1$, the scheme is in a semi-implicit form. For $0.5 \leq \theta_{1} \leq 1$ and $\theta_{2}=0$, the scheme is in an explicit form.

Defining $\boldsymbol{F}=\boldsymbol{u} \otimes \boldsymbol{u}, \boldsymbol{G}=-\boldsymbol{\tau} / \rho, Q=-\boldsymbol{f}_{b}$, Eq. (20) can then be written in the form of Eq. (15). Then it can be discretized using the algorithm given in Section 3.1.

$$
\frac{\Delta \boldsymbol{u}}{\Delta t}+\nabla \cdot \boldsymbol{F}+\nabla \cdot \boldsymbol{G}+Q+\frac{1}{\rho} \nabla p^{n+\theta_{2}}=0
$$

The time discretization of the above equation along the characteristic gives

$$
\Delta \boldsymbol{u}=-\Delta t\left(\nabla \cdot \boldsymbol{F}+\nabla \cdot \boldsymbol{G}+Q+\frac{1}{\rho} \nabla p^{n+\theta_{2}}\right)^{n}+\frac{\Delta t^{2}}{2} \boldsymbol{u} \cdot \nabla\left(\nabla \cdot \boldsymbol{F}+Q+\frac{1}{\rho} \nabla p^{n+\theta_{2}}\right)^{n}
$$

According to the basic idea of the fractional step procedure, $\Delta \boldsymbol{u}$ is divided into two parts

$$
\Delta \boldsymbol{u}=\Delta \boldsymbol{u}^{*}+\Delta \boldsymbol{u}^{* *}
$$

The intermediate velocity increment $\Delta \boldsymbol{u}^{*}$ and the velocity correction increment $\Delta \boldsymbol{u}^{* *}$ can be written as follows.

$$
\begin{gathered}
\Delta \boldsymbol{u}^{*}=-\Delta t(\nabla \cdot \boldsymbol{F}+\nabla \cdot \boldsymbol{G}+Q)^{n}+\frac{\Delta t^{2}}{2} \boldsymbol{u} \cdot \nabla(\nabla \cdot \boldsymbol{F}+Q)^{n} \\
\Delta \boldsymbol{u}^{* *}=-\Delta t \frac{1}{\rho} \nabla p^{n+\theta_{2}}+\frac{\Delta t^{2}}{2} \boldsymbol{u} \cdot \nabla\left(\frac{1}{\rho} \nabla p^{n+\theta_{2}}\right)
\end{gathered}
$$

The intermediate velocity increment $\Delta \boldsymbol{u}^{*}$ is calculated explicitly in the first step where the influence of the pressure gradient term is not involved. Once the pressure increment $\Delta p^{n}$ is obtained according to intermediate velocity, the velocity in the next time step $\boldsymbol{u}^{n+1}$ can be calculated after the velocity correction value $\Delta \boldsymbol{u}^{* *}$ is determined by $\Delta p^{n}$.The whole process is described in detail as follows.

Step 1: Intermediate velocity calculation

$\Delta \boldsymbol{u}^{*}$ can be calculated by discretizing Eq. (24) based on the standard Galerkin approximation. The discretization format is

$$
\begin{aligned}
\int \boldsymbol{N}^{T} \boldsymbol{N} d \Omega \Delta \overline{\boldsymbol{u}}^{*}= & -\Delta t\left[\int \boldsymbol{N}^{T}(\nabla \cdot \boldsymbol{F}+Q) d \Omega-\int \nabla \boldsymbol{N}^{T} \boldsymbol{G} d \Omega\right] \\
& -\frac{\Delta t^{2}}{2} \int \nabla \cdot\left(\boldsymbol{N}^{T} \boldsymbol{u}\right)(\nabla \cdot \boldsymbol{F}+Q) d \Omega-\Delta t \int \boldsymbol{N}^{T} \boldsymbol{G} \cdot \boldsymbol{n} d \Gamma
\end{aligned}
$$

Step 2: Pressure calculation 
To calculate the pressure, $\boldsymbol{u}^{n+\theta_{1}}=\boldsymbol{u}^{n}+\theta_{1} \Delta \boldsymbol{u}^{*}+\theta_{1} \Delta \boldsymbol{u}^{* *}$ is substituted into Eq. (19) and a Poisson equation is obtained

$$
\nabla \cdot\left(\boldsymbol{u}^{n}+\theta_{1} \Delta \boldsymbol{u}^{*}-\theta_{1} \Delta t \frac{1}{\rho} \nabla p^{n+\theta_{2}}\right)=0
$$

Eq. (27) can also be discretized with the standard Galerkin approximation because of the self-adjoint property of $\Delta p$ and the discretization format is

$$
\begin{aligned}
\theta_{1} \theta_{2} \Delta t \int \frac{1}{\rho} \nabla \boldsymbol{N}^{T} \cdot \nabla \boldsymbol{N} d \Omega \Delta \bar{p}^{n} & =-\theta_{1} \Delta t \oint \frac{1}{\rho} \boldsymbol{N}^{T} \nabla p^{n+\theta_{2}} \cdot \boldsymbol{n} d \Gamma+\theta_{1} \Delta t \int \frac{1}{\rho} \nabla \boldsymbol{N}^{T} \cdot \nabla p^{n} d \Omega \\
& +\int \boldsymbol{N}^{T} \nabla \cdot\left(\boldsymbol{u}^{n}+\theta_{1} \Delta \boldsymbol{u}^{*}\right) d \Omega
\end{aligned}
$$

The third term on the right hand side of Eq. (28) is integrated by parts obtaining

$$
\begin{aligned}
\theta_{1} \theta_{2} \Delta t \int \frac{1}{\rho} \nabla \boldsymbol{N}^{T} \cdot \nabla \boldsymbol{N} d \Omega \Delta \bar{p}^{n} & =\int \nabla \boldsymbol{N}^{T} \cdot\left[\left(\boldsymbol{u}^{n}+\theta_{1} \Delta \boldsymbol{u}^{*}\right)-\theta_{1} \Delta t \frac{1}{\rho} \nabla p^{n}\right] d \Omega \\
& -\oint \boldsymbol{N}^{T}\left[\left(\boldsymbol{u}^{n}+\theta_{1} \Delta \boldsymbol{u}^{*}\right)-\theta_{1} \Delta t \frac{1}{\rho} \nabla p^{n+\theta_{2}}\right] \cdot \boldsymbol{n} d \Gamma
\end{aligned}
$$

Neglecting high order terms, Eq. (30) can be obtained according to Eq. (25).

$$
\left(\boldsymbol{u}^{n}+\theta_{1} \Delta \boldsymbol{u}^{*}\right)-\theta_{1} \Delta t \frac{1}{\rho} \nabla p^{n+\theta_{2}}=\boldsymbol{u}^{n}+\theta_{1} \Delta \boldsymbol{u}^{*}+\theta_{1} \Delta \boldsymbol{u}^{* *}=\boldsymbol{u}^{n+\theta_{1}}
$$

As a result, the second term on the right hand side of Eq. (29) can be written as

$$
\oint \boldsymbol{N}^{T}\left[\left(\boldsymbol{u}^{n}+\theta_{1} \Delta \boldsymbol{u}^{*}\right)-\theta_{1} \Delta t \frac{1}{\rho} \nabla p^{n+\theta_{2}}\right] \cdot \boldsymbol{n} d \Gamma=\oint \boldsymbol{N}^{T} \boldsymbol{u}^{n+\theta_{1}} \cdot \boldsymbol{n} d \Gamma
$$

It is observed that the determination of the term $\nabla p^{n+\theta_{2}}$ is avoided on the boundary when the normal velocity on the boundary is known. This kind of boundary conditions include inflow, slip and non-slip boundary conditions.

If the normal velocity on the boundary is unknown, for example, on the boundary where the outflow condition or rigid-lid assumption is adopted, $\nabla p^{n+\theta_{2}}$ is assumed to be $\nabla p^{n}$.

Step 3: Velocity correction

By taking the effect of pressure into consideration, the velocity increment is modified. The discretization format of the velocity correction term is

$$
\begin{aligned}
\int \boldsymbol{N}^{T} \boldsymbol{N} d \Omega \Delta \overline{\boldsymbol{u}}^{* *} & =-\Delta t \int \frac{1}{\rho} \boldsymbol{N}^{T} \nabla\left(p^{n}+\theta_{2} \Delta p^{n}\right) d \Omega \\
& -\frac{\Delta t^{2}}{2} \int \nabla \cdot\left(\boldsymbol{N}^{T} \boldsymbol{u}\right) \frac{1}{\rho} \nabla\left(p^{n}+\theta_{2} \Delta p^{n}\right) d \Omega
\end{aligned}
$$




\subsection{Discretization of the ICLS functions}

The equations used to implement the ICLS method for $n$-phases fluids, namely Eq. (6), Eq. (7), Eq. (11) and Eq. (12), can be discretized by substituting the corresponding $\boldsymbol{F}, \boldsymbol{G}$ and $Q$ into Eq. (18) The relevant expressions for those three terms are as follows.

For Eq. (6), $\boldsymbol{F}=\boldsymbol{u} H, \boldsymbol{G}=Q=0$

For Eq. (7), $\boldsymbol{F}=H(1-H) \boldsymbol{n}, \boldsymbol{G}=-\varepsilon(\nabla H \cdot \boldsymbol{n}) \boldsymbol{n}, Q=0$

For Eq. (11), $\boldsymbol{F}=\boldsymbol{u} \psi, \boldsymbol{G}=Q=0$

For Eq. (12), $\boldsymbol{F}=\tilde{\boldsymbol{u}} \psi, \boldsymbol{G}=0, Q=-S\left(\psi^{n}\right)-\psi \nabla \cdot \tilde{\boldsymbol{u}}$

\section{Verification and application}

The three-phases Zalesak slotted disk test case is simulated to prove the conservative feature of the proposed method. To verify the correctness of the proposed method in dealing with the actual engineering problems, this method is applied to model landslide-generated waves at the Lituya Bay. The outcomes are compared with the results of existing publications. In addition, this method is also applied to model the impulse waves in the Jinsha River caused by the Halaowo landslide and results are used to estimate the effects of the waves on the Xiangjiaba dam, which is a practical hydraulic project in the southwest of China.

\subsection{Three-phases Zalesak slotted disk}

The benchmark of Zalesak slotted disk is widely applied to test the advection ability of interface capturing methods and to characterize how accurately sharp corners are transported. However, most tests are studied for two-phases problems. This test case is now expanded to clarify the conservation feature of the ICLS method for three-phases flows. The Zalesak slotted disk is defined in a calculation domain $x \in[0,1], y \in[0,1]$. The initial center of the disk is located at $(0.50,0.75)$ with a radius $R=0.15$. The width and depth of the slot is 0.05 and 0.25 respectively. The whole domain is filled with three kinds of fluids and is discretized with $400 \times 400$ quadrilateral elements. To give a clear definition of the domains for these three-phases flows, $\mathrm{D}$ specifies the whole calculation domain while $\mathrm{D}_{\mathrm{d}}$ is used to represent the domain of the slotted disk. As a result, the three domains at the initial time $t=0$ can be defined as

$$
\begin{aligned}
& \mathrm{D}_{1}=\left\{(x, y) \in \mathrm{D}-\mathrm{D}_{\mathrm{d}}\right\} \\
& \mathrm{D}_{2}=\left\{(x, y) \in\left(\mathrm{D}_{\mathrm{d}} \cap(y<0.75)\right)\right\} \\
& \mathrm{D}_{3}=\left\{(x, y) \in\left(\mathrm{D}_{\mathrm{d}} \cap(y \geq 0.75)\right)\right\}
\end{aligned}
$$


Moreover, two hyperbolic tangent functions are applied to represent the interfaces. Initially, the functions are defined as

If $(x, y) \in \mathrm{D}_{2}+\mathrm{D}_{3}$, then $0 \leq H_{1}<0.5$. If $(x, y) \in \mathrm{D}_{1}$, then $0.5 \leq H_{1} \leq 1$.

If $(x, y) \in \mathrm{D}_{3}$, then $0 \leq H_{2}<0.5$. If $(x, y) \in \mathrm{D}_{1}+\mathrm{D}_{2}$, then $0.5 \leq H_{2} \leq 1$.

The slotted disk is rotated according to the following equations

$$
\begin{aligned}
& u(x, y)=-2 \pi(y-0.5) \\
& v(x, y)=2 \pi(x-0.5)
\end{aligned}
$$

which represents a rigid body rotation with respect to $(0.5,0.5)$. The disk completes one revolution after one unit of time. The total calculation time is 2 , i.e. two revolutions.

The evolution of the disk in the first two revolutions is shown in Fig. 2. As expected, the interface after two revolutions is nearly identical to the initial shape with negligible numerical diffusion. The original sharp corners of the disk are slightly smoothed in this simulation because the interfaces are smeared in a spreading width $\varepsilon$, which is twice as long as the grid length. This kind of smoothness can only be observed during the beginning period. Afterwards, the interfaces become stable and changeless. 


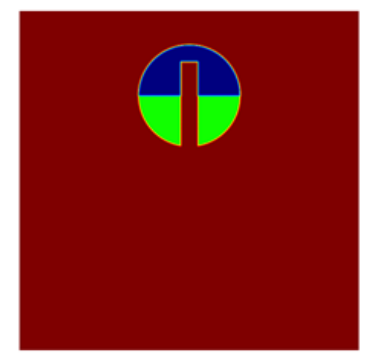

$t=0$

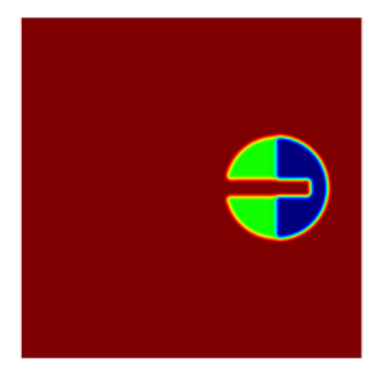

$t=0.75$

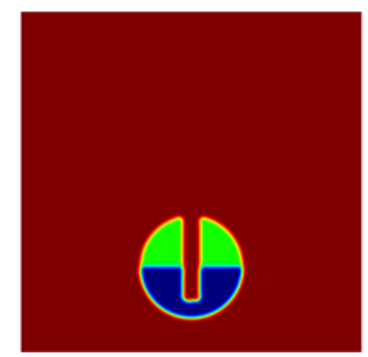

$t=1.5$

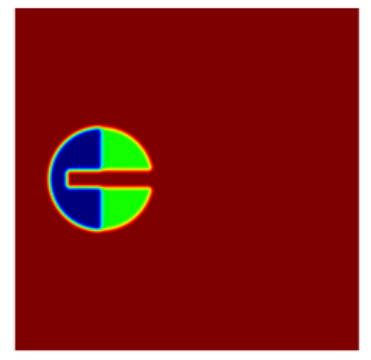

$t=0.25$

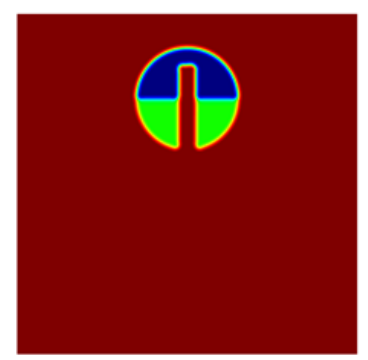

$t=1$

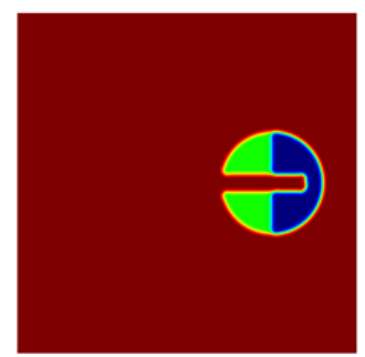

$t=1.75$

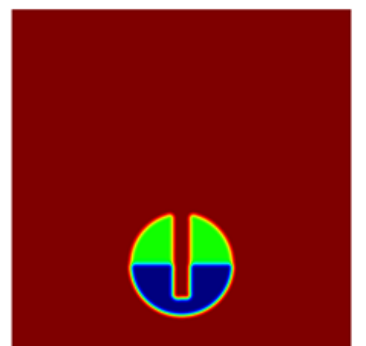

$t=0.5$

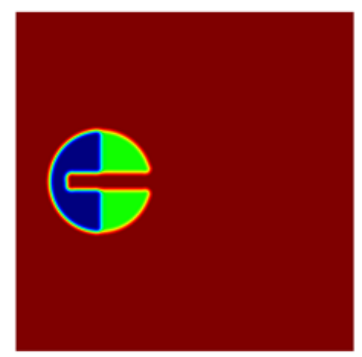

$$
t=1.25
$$

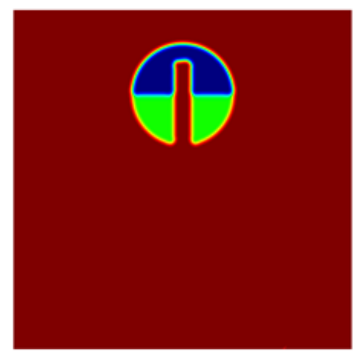

$t=2$

Fig. 2 Two complete revolutions of the disk.

Even though the corners are not as sharp as in the original condition, mass is still conserved. To further describe the conservation feature of the current method, two parameters named the percentage of relative mass conservation errors $e_{r 1}(t)$ and $e_{r 2}(t)$ are employed. The definition of $e_{r 1}(t)$ is

$$
e_{r 1}(t)=\left(1-A_{1} / A_{o 1}\right) \times 100
$$

in which $A_{1}=\int H_{1}(x, y) d \Omega$ for the area which satifies $0 \leq H_{1}<0.5$ at arbitrary time $t$ and $A_{o 1}$ is the area at the initial time $t=0$.

Similarly, $e_{r 2}(t)$ is defined as

$$
e_{r 2}(t)=\left(1-A_{2} / A_{o 2}\right) \times 100
$$

in which $A_{2}=\int H_{2}(x, y) d \Omega$ for the area which satisfies $0 \leq H_{2}<0.5$ at arbitrary time $t$ and $A_{o 2}$ is the area at the initial time $t=0$. 


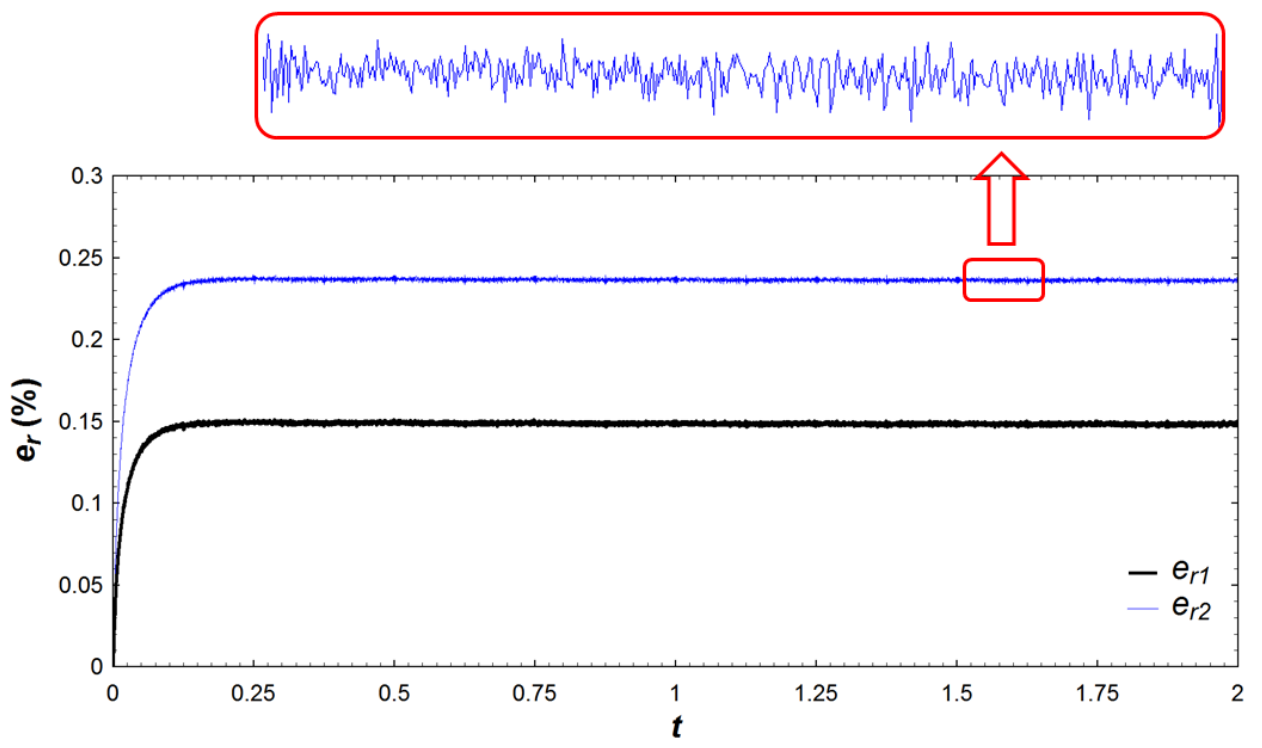

Fig. 3 Percentage of relative mass conservation errors versus time.

The relative global mass conservation errors are plotted in Fig. 3. It can be observed that the errors increase slightly in the initial stage, from $t=0$ to $t=0.15$, then they stay relatively stable and fluctuate around certain values. The initial increasement is induced by the instantaneous increasing thickness of the interface. The fluctuation is caused by the integral error as the mass is integrated in an element using the values on Gaussian points and this deviation is random, which can be seen in the local zoom area in Fig. 3. Both $e_{r 1}(t)$ and $e_{r 2}(t)$ are smaller than $0.25 \%$ after two revolutions, which is similar as the errors after one revolution. Most of the derivation can be avoided if an initial thickness of the interface is set up and the remaining error is small, random and fluctuant. This verifies that the current method is stable and conservative.

\subsection{Lituya Bay impulse wave analysis}

The 1958 landslide-generated wave at Lituya Bay attracts the attention of many researchers and abundant studies have been carried out around this topic until now [30, 4952]. The layout of the Lituya Bay is shown in Fig. 4. 


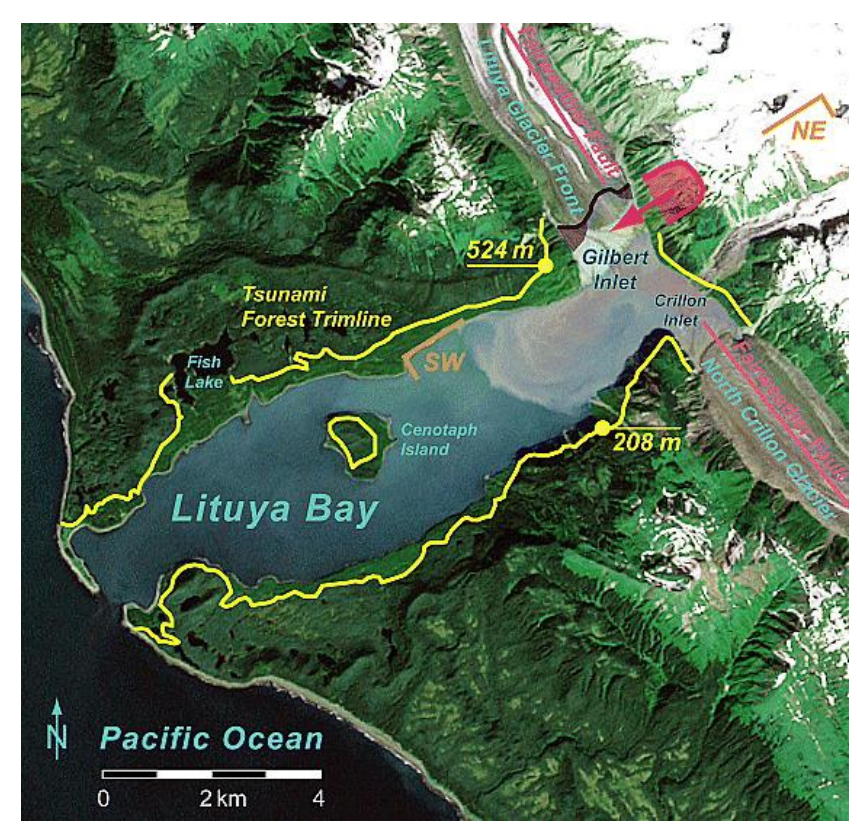

Fig. 4 Photo of the T-shaped Lituya Bay (from Weiss et al. [52]).

A two-dimensional physical model built by Fritz et al. [49] is widely used in numerical simulations, shown in Fig. 5. This model was set up with a scale 1: 675. The landslide mass (with a volume per unit width of $37.2 \times 10^{3} \mathrm{~m}^{3} / \mathrm{m}$ ) was modelled as an artificial granular material and the bulk density was set to be $1.61 \mathrm{t} / \mathrm{m}^{3}$ with a porosity of $39 \%$. The effective internal friction angle was $43^{\circ}$. The mean impact velocity of the mass was $110 \mathrm{~m} / \mathrm{s}$, which was estimated by the free fall equation at the slide centroid (with an elevation of $610 \mathrm{~m}$ ).

In our simulation, both the physical model and the parameters of landslide suggested by Fritz et al. [49] are adopted. The computation domain is meshed with three nodes triangular elements and the number of elements in the whole domain is 94758. Non-friction and impermeable walls are used as boundary conditions. 


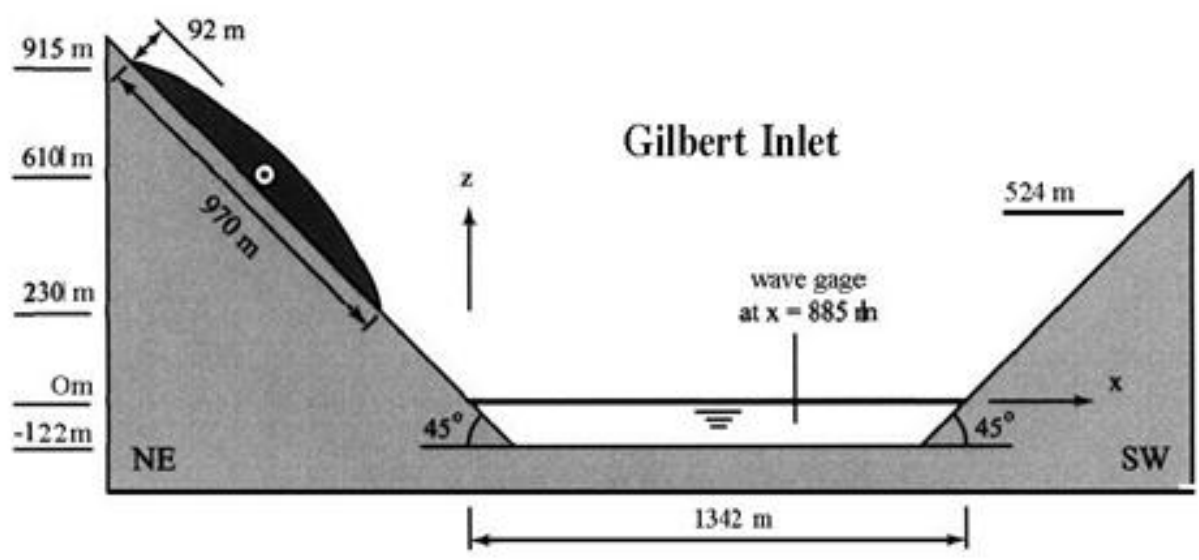

Fig. 5 Cross section of the simplified inlet used in the current method (from Fritz et al. [49]).

Fig. 6 presents the density images in our simulation. The series of figures show the whole process namely the generation, propagation and run-up of waves, along with the formation and breaking of air cavity.

The slide begins to penetrate into the water in Fig. 6a and energy transforms from the slide to the water body. In the next instant, slide bulking, which can also be called the impact crater, is formed and the water body is separated on the shoulder of the landslide mass. The height of the wave keeps increasing as the size of the slide bulking expands (Fig. 6b, c) because the slide penetrates into the water with a high speed. The water reattaches to the bottom and a cavity filled with air can be observed behind the slide because the slide accelerates and its velocity is larger than the wave velocity (Fig. 6d, e). The landslide mass continues sliding along the bottom and eventually deposits. As a result, the air cavity collapses and a strong mixing between the water and air can be observed. Afterwards, both the landslide mass and the wave run up to the opposite bank (Fig. 6f, g, h). The main body of the wave already leaves the mixing area before the air cavity collapses, therefore, the leading wave is little influenced by the phase mixing. As a result, the property of the wave is almost not affected by energy losses due to the air entrainment. 


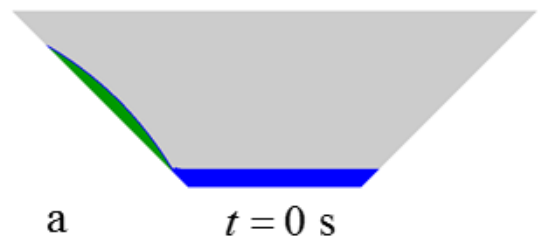

b

$t=5.2 \mathrm{~s}$
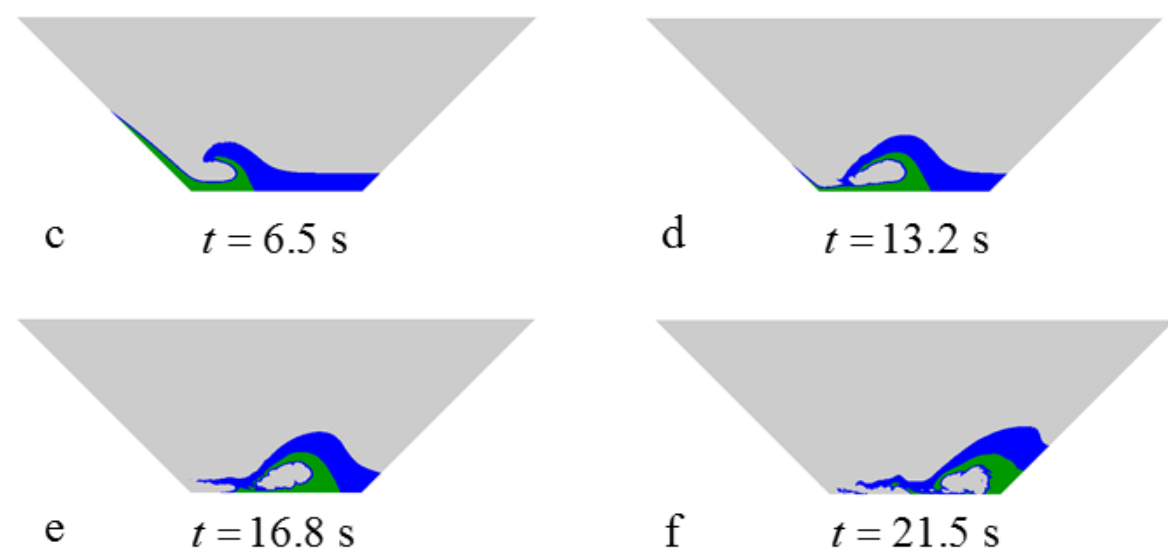

Air

Water

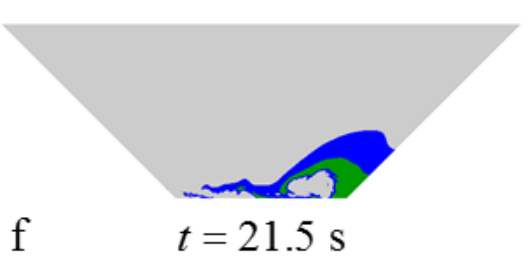

Landslide

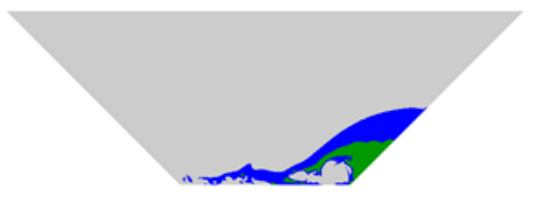

g $\quad t=23.6 \mathrm{~s}$

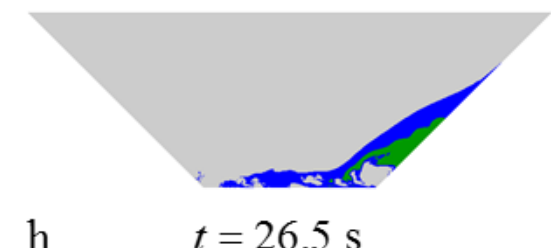

Fig. 6 Evolution of waves generated by the Lituya Bay landslide.

The current outputs are compared with the existing results, shown in Table 2. In this table, $\rho, \rho_{\text {bulk }}, n_{\text {por }}$ and $\phi$ represent the particle density, bulk density, porosity and internal friction angle of landslides, respectively. $h_{\max }$ and $h_{r u n}$ stand for the maximum water surface elevation at $885 \mathrm{~m}$ and runup height.

\begin{tabular}{lccccccc}
\hline Method & Source & $\rho\left(\mathrm{t} / \mathrm{m}^{3}\right)$ & $\rho_{\text {bulk }}\left(\mathrm{t} / \mathrm{m}^{3}\right)$ & $n_{\text {por }}(\%)$ & $\phi\left(^{\circ}\right)$ & $h_{\max }(\mathrm{m})$ & $h_{\text {run }}(\mathrm{m})$ \\
\hline Field data & {$[50]$} & 2.7 & - & - & - & 150 & 524 \\
\hline $\begin{array}{l}\text { Physical } \\
\text { experiment }\end{array}$ & {$[49]$} & 2.64 & 1.61 & 39 & 43 & 152 & 526 \\
\hline Numerical & {$[30]$} & - & 1.6 & 39 & 0 & 266 & - \\
simulation & - & 1.6 & 39 & 15 & 226 & - \\
\hline $\begin{array}{l}\text { Numerical } \\
\text { simulation }\end{array}$ & current & - & 1.61 & 39 & 43 & 173 & 569 \\
\hline
\end{tabular}

Table 2 Parameters and results of different methods. 
With the application of the present method, both the maximum wave height in a certain gauge point and the runup are larger than the results of the physical experiment. The same phenomenon can also be observed in [30]. Compared with the experimental results, the current results are exaggerated because of the different constitutive models of the landslide and the scale effect. The landslide is treated as a fluid-like material in the current simulation, while it was modelled as a granular flow in the experiment. Actually, the property of the landslide may be between these two conditions. Considering the high moisture content and thick alluvium deposits of slopes in reservoirs, it is appropriate to treat this kind of landslides as fluid-like when they slide with a high speed, but the difference in material could contribute to the overprediction. It should be noticed that the results provided by the current method are closer to the outputs of both the field data and physical experiment, compared with the results in [30]. Hence this proposed method can be used to capture the whole process of landslide waves, including generation, propagation and run-up.

\subsection{Application to the Xiangjiaba Dam impulse wave}

The Xiangjiaba hydropower station is located on the lower reaches of the Jinsha River, which is on the Yunnan province boundary with Sichuan province. This hydropower station is the last step of the power stations construction on the Jinsha River and plays a vital role in the west to east electricity transmission project.

Various factors including heavy rainfall, freeze-thaw cycles and pore water pressure changes, may attribute to the instability of existing slopes. Once these large landslides slip into the Jinsha River, the generated impulse waves will impose a threat to the residents along the shoreline and the Xiangjiaba hydropower station. One of the most dangerous instable slopes named Halaowo (around $7.26 \times 10^{6} \mathrm{~m}^{3}$ ) is located upstream of the Xiangjiaba Dam (with an elevation of $384 \mathrm{~m}$ ) with a horizontal distance of $18 \mathrm{~km}$. The topographic map of the river channel is given in Fig. 7 based on a digital elevation model (DEM) with a resolution of $2.5 \mathrm{~m}$. The still water level of the Jinsha River is $380 \mathrm{~m}$. 


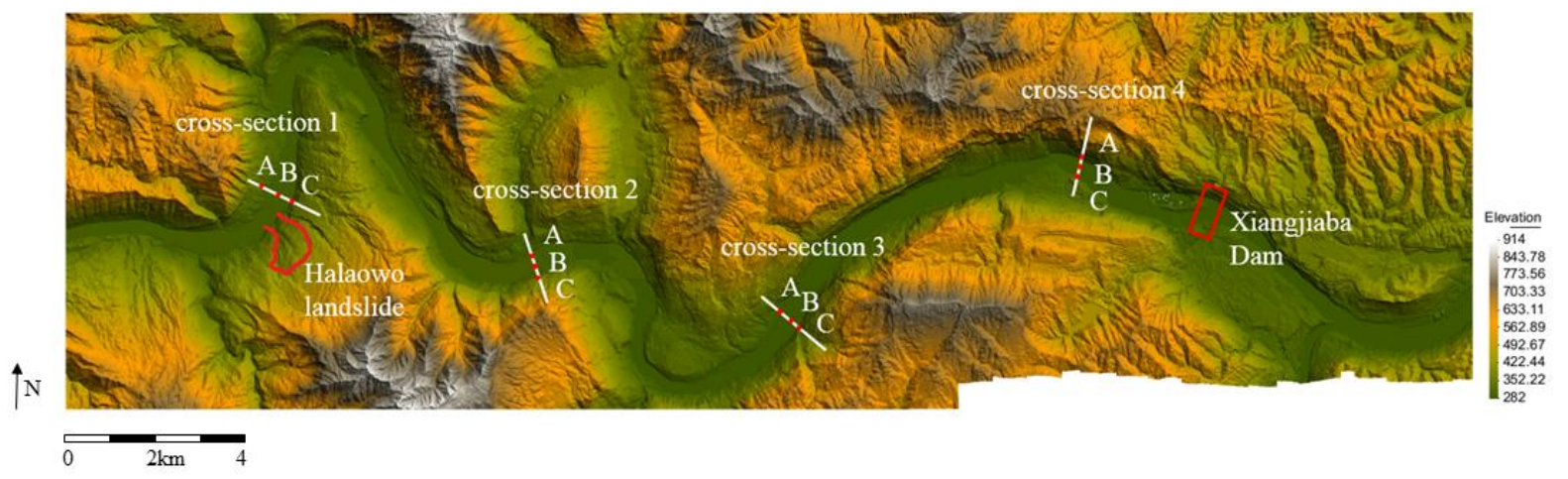

Fig. 7 DEM of the Halaowo landslide and the Xiangjiaba Dam: on the left side the Halaowo landslide is shown, on the right side the Xiangjiaba Dam, the solid lines are cross sections, and points on the solid lines specify the gauge locations.

To simulate the generation and propagation of waves, the following parameters are used. The bulk density of the Halaowo landslide is $2200 \mathrm{~kg} / \mathrm{m}^{3}$, which is within the range expected in the field situation. The Bingham model is used with the yield stress $\tau_{y 0}=1 \mathrm{kPa}$ and the internal friction angle $\phi=25^{\circ}$. Non-slip and impermeable walls are adopted as the boundary conditions.

According to the DEM, the three dimensional model of the Halaowo landslide is established, shown in Fig. 8. In this figure, the Y direction points toward north. The landslide mass is partly submerged and the center of sliding mass is above the water surface. The computation domain is meshed with 207081 four nodes tetrahedral elements.

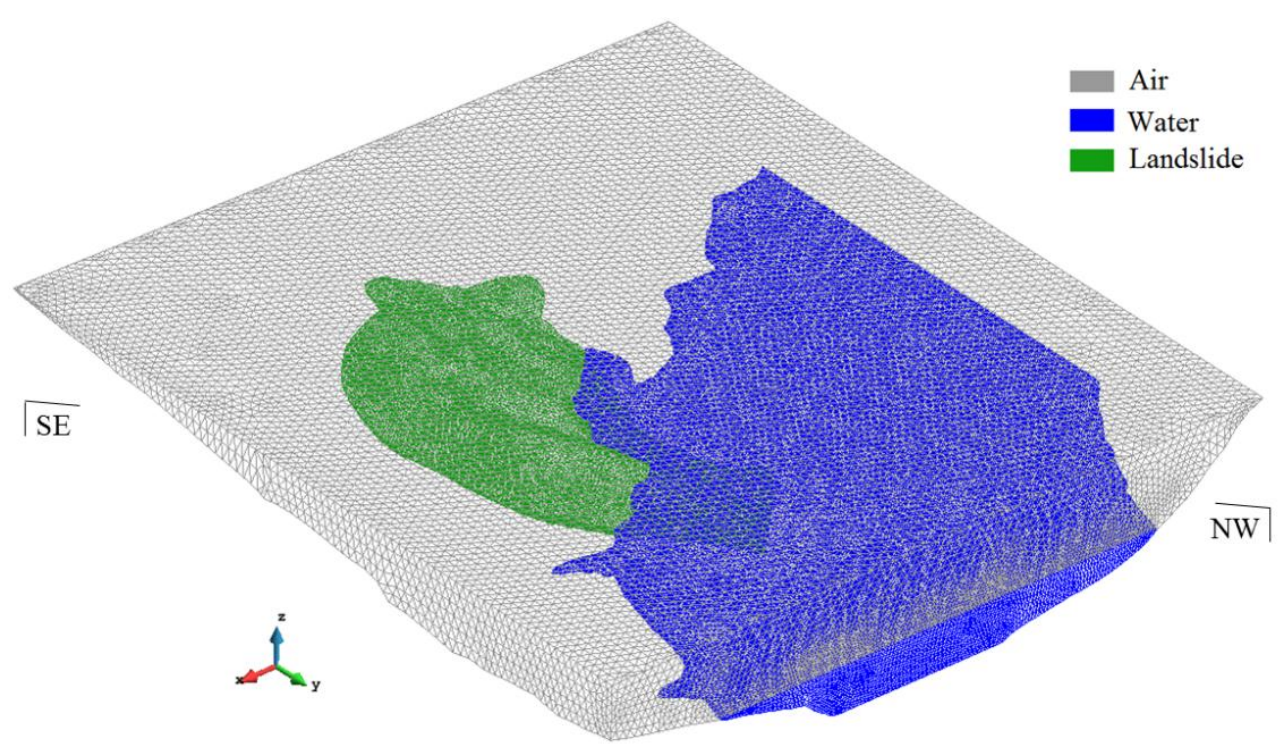

Fig. 8 Three dimensional model of the Halaowo landslide. 
To clarify the three-phases domain which includes the typical section, a twodimensional plan is shown in Fig. 9. This cross section is also indicated in Fig. 8.

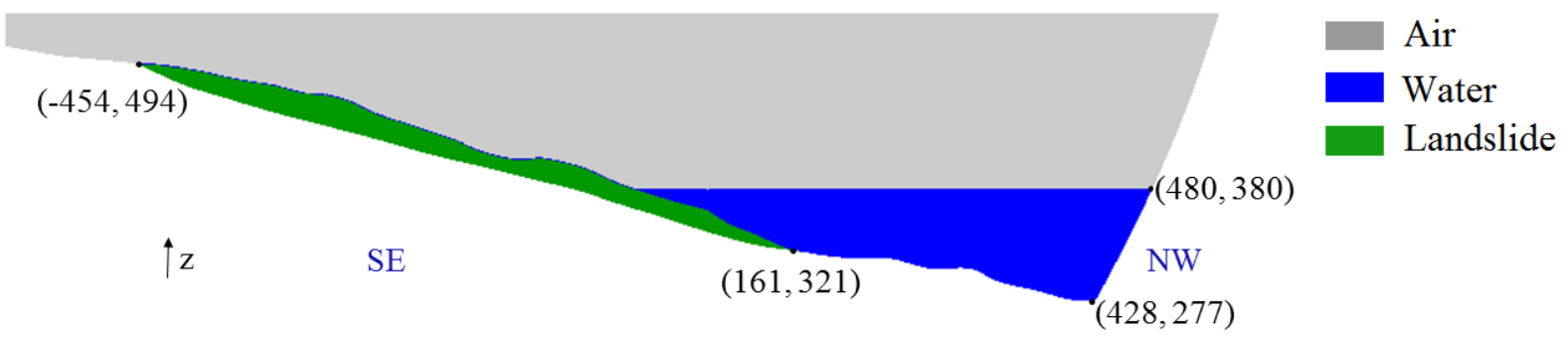

Fig. 9 Plan including the typical section of the landslide. The unit of the coordinates is $\mathrm{m}$.

The density contours on the typical section at $t=0 \mathrm{~s}, t=3 \mathrm{~s}, t=9 \mathrm{~s}, t=13 \mathrm{~s}, t=16 \mathrm{~s}$ and $t=17 \mathrm{~s}$ have been plotted in Fig. 10. The water surface height of the impulse wave reaches the maximum value $44.27 \mathrm{~m}$ at $t=16 \mathrm{~s}$. After reaching the maximum value, the wave runs up on the opposite bank and reflection can be observed. At that moment, almost the entire landslide mass has entered the water body.

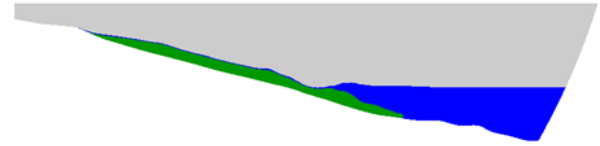

$t=0 \mathrm{~s}$

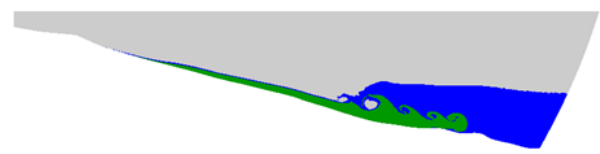

$t=9 \mathrm{~s}$

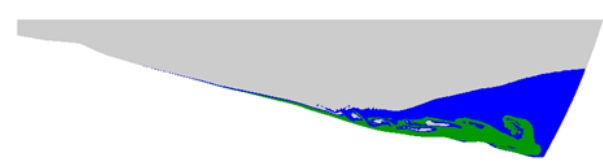

$t=16 \mathrm{~s}$

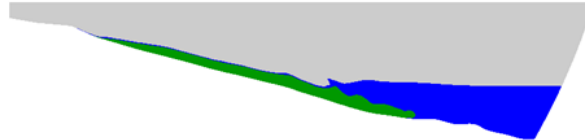

$t=3 \mathrm{~s}$

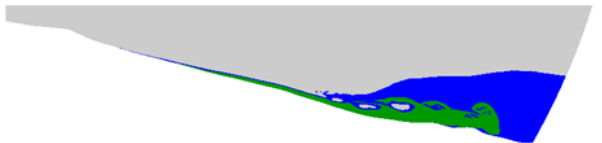

$t=13 \mathrm{~s}$

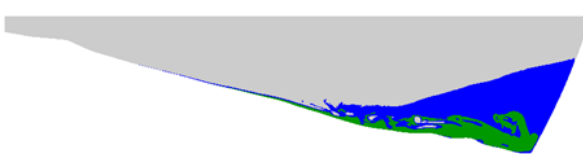

$t=17 \mathrm{~s}$
Air

Water

Landslide

Fig. 10 Evolution of waves generated by the Halaowo landslide at cross-section 1 .

A Kelvin-Helmholtz type instability can be observed in Fig. 10. This instability phenomenon has also been found and reported in the study of Rzadkiewicz et al. [53] who carried out an experiment about the sliding process of coarse gravel. When the Halaowo landslide slides down with a relatively high velocity, the velocity gradient at the interface is large. Hence, the large gradient of velocity leads to a strong shearing effect on the waterbody, which causes Kelvin-Helmholtz instabilities. 
After the impulse wave is generated, it propagates to both upstream and downstream sides. To present the generation and propagation of the landslide wave, twelve gauging points are selected on four sections, as shown in Fig. 7. The water levels are plotted in Fig. 11 over time.
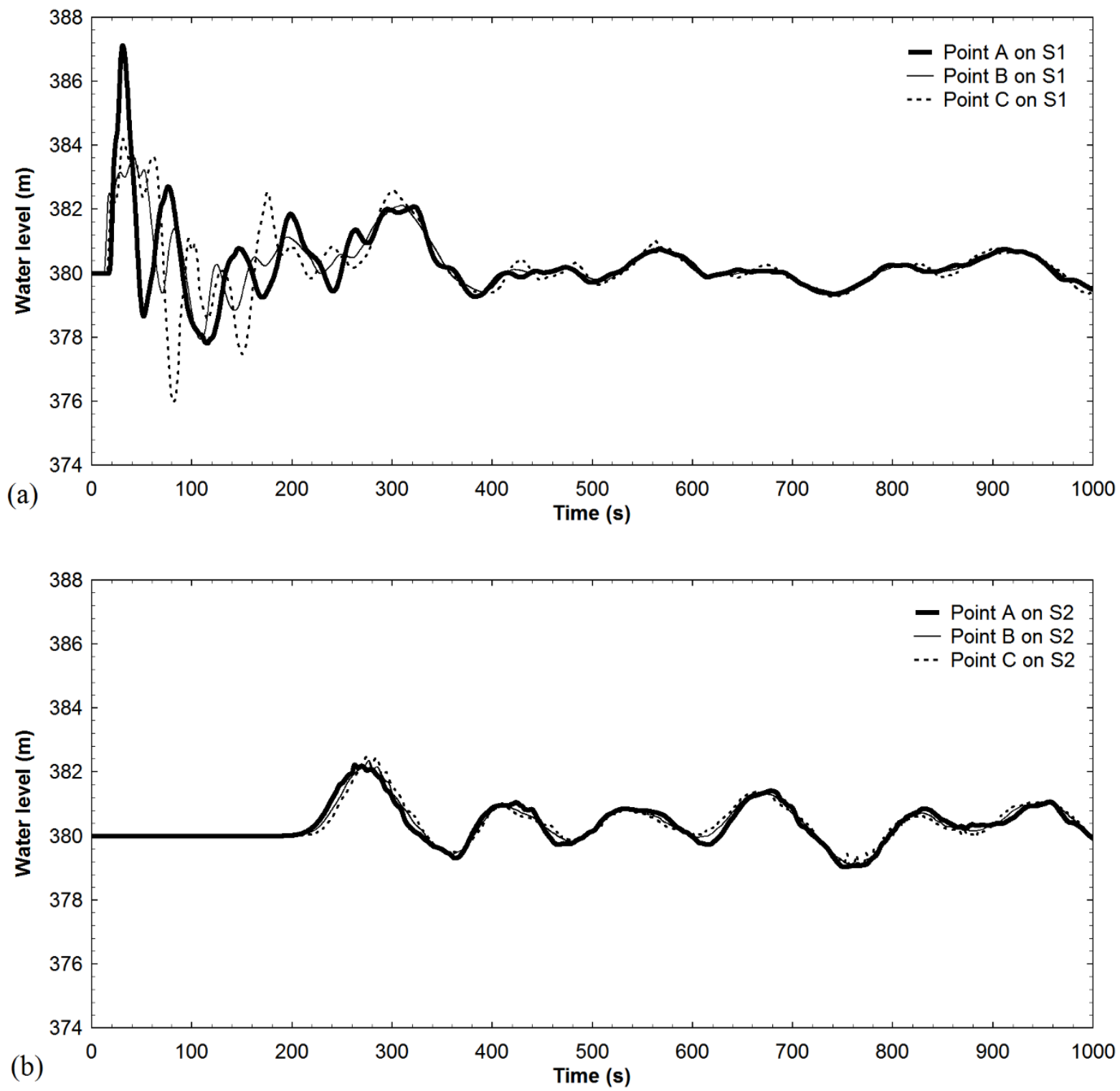

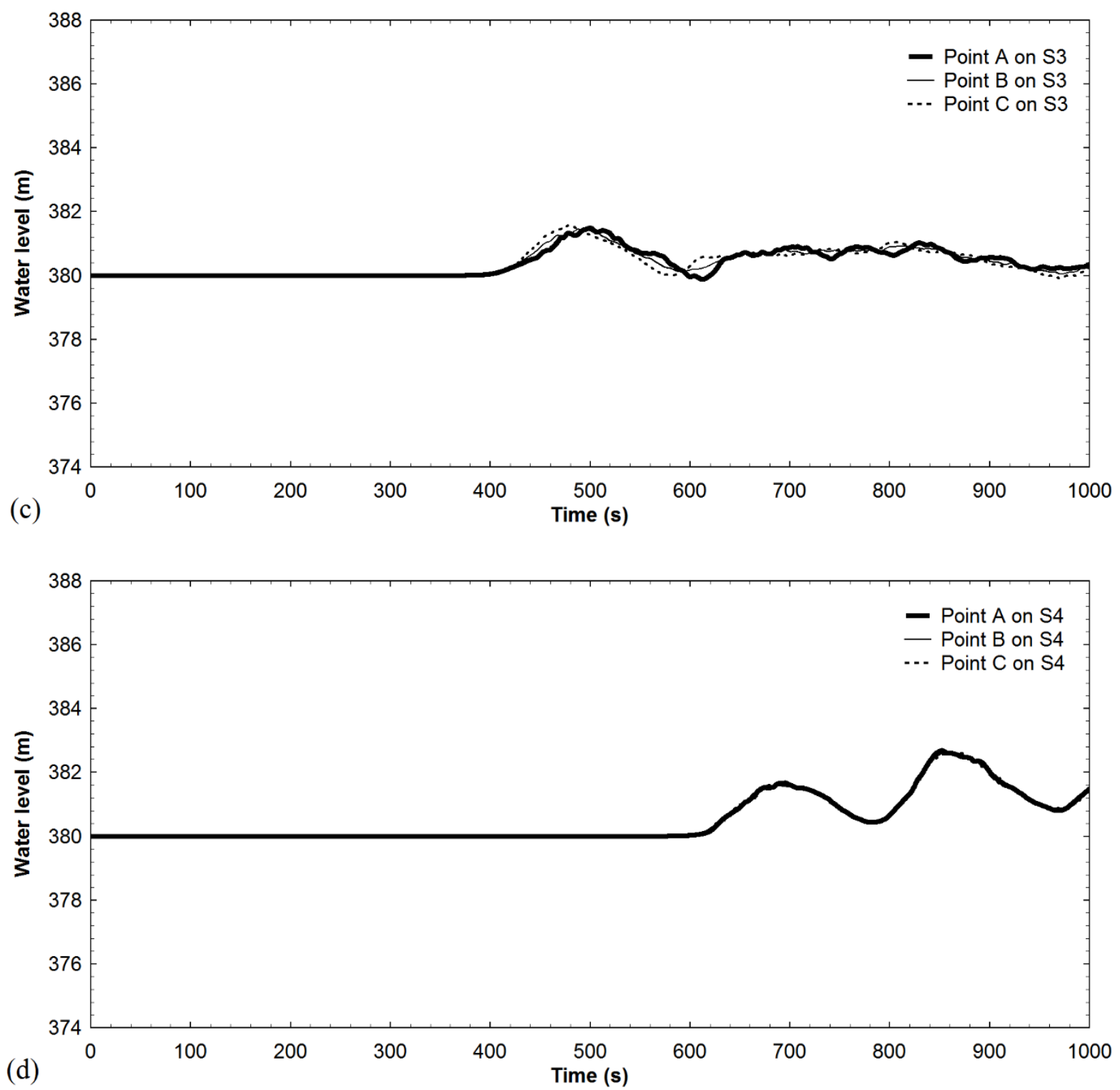

Fig. 11 Water levels versus time at twelve gauging points (a) value of water level on section 1. (b) value of water level on section 2. (c) value of water level on section 3. (d) value of water level on section 4 .

Since section 1 is close to the position of the Halaowo landslide, the water level-time variation curves of three measure points are not similar with each other in the initial stage. The maximum water level, $387.2 \mathrm{~m}$, can be found at point $\mathrm{A}$ at $t=30 \mathrm{~s}$, while the initial water levels at point $\mathrm{B}$ and point $\mathrm{C}$ are significantly smaller. This phenomenon can be attributed to different positions of these three points. It can be observed from Fig. 7 that point $\mathrm{A}$ is on the opposite shore of the landslide, while point $\mathrm{B}$ and point $\mathrm{C}$ are at the river centre and on the same side with the slide, respectively. Wave run-up occurs at point A leading to a higher water level. As time goes on, water levels of different points in section 1 converge 
toward uniformity. The physical parameters at the three points in section 2 are similar with each other and this phenomenon can also be observed at section 3. At $t=270 \mathrm{~s}$, the maximum wave crest propagates to section 2 and at $t=500 \mathrm{~s}$, it arrives at section 3 . The maximum water level is $382.5 \mathrm{~m}$ and $381.5 \mathrm{~m}$, respectively. The wave height attenuation is obvious during the propagation process because of the dissipation of energy. At $t=700 \mathrm{~s}$, the wave reaches section 4 and the maximum water level in the second period is $382.7 \mathrm{~m}$, which is larger than that in section 3 due to reflections from the dam. Even though the dam is not reproduced in this simulation, a non-slip and impermeable boundary condition is employed at the position of the dam. As a result, reflections can still be observed. The whole propagation process can be observed from Fig. 10a- Fig. 10d which represent the water level at selected moments in time $t=30 \mathrm{~s}, t=270 \mathrm{~s}, t=500 \mathrm{~s}, t=700 \mathrm{~s}$ respectively.
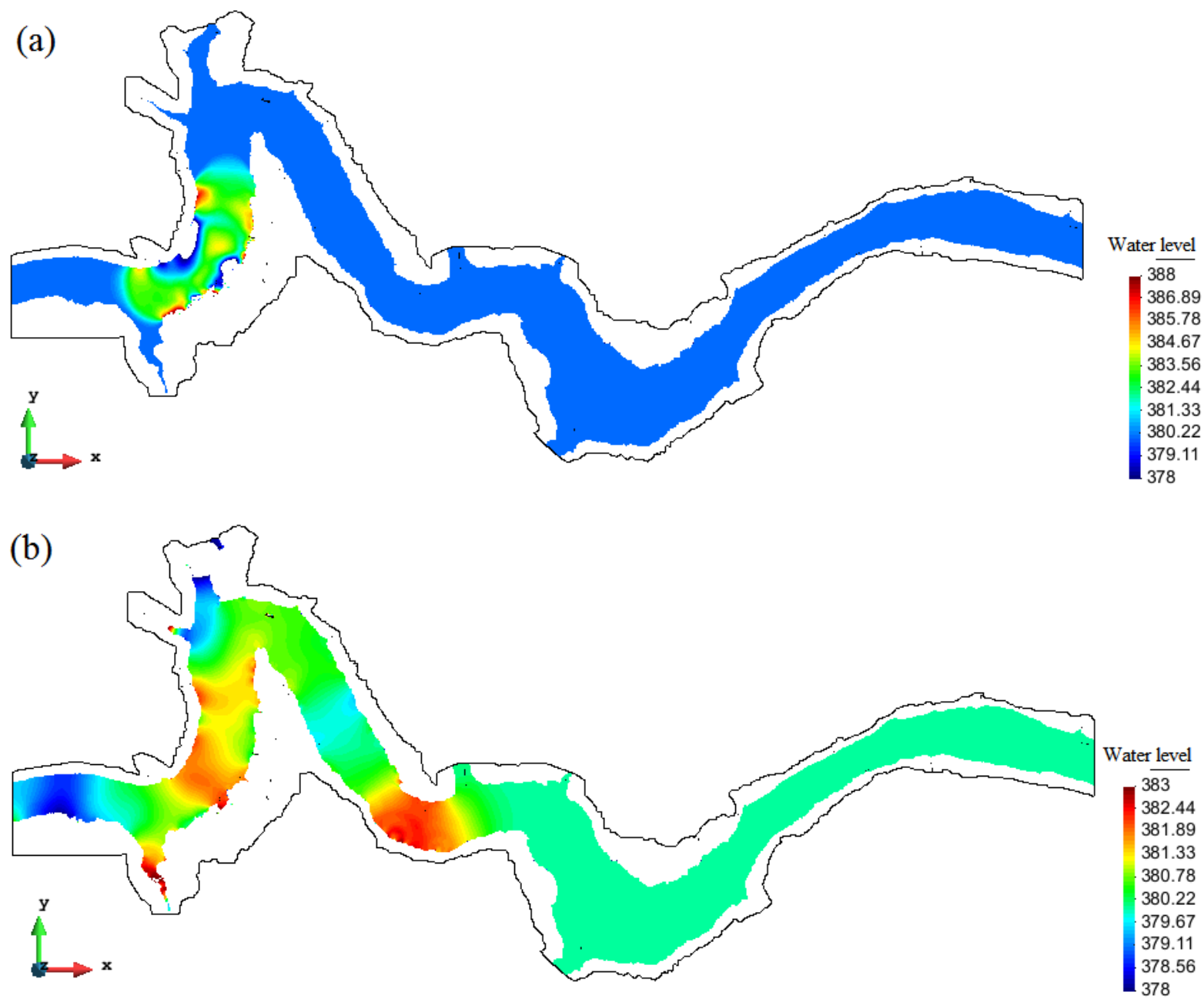

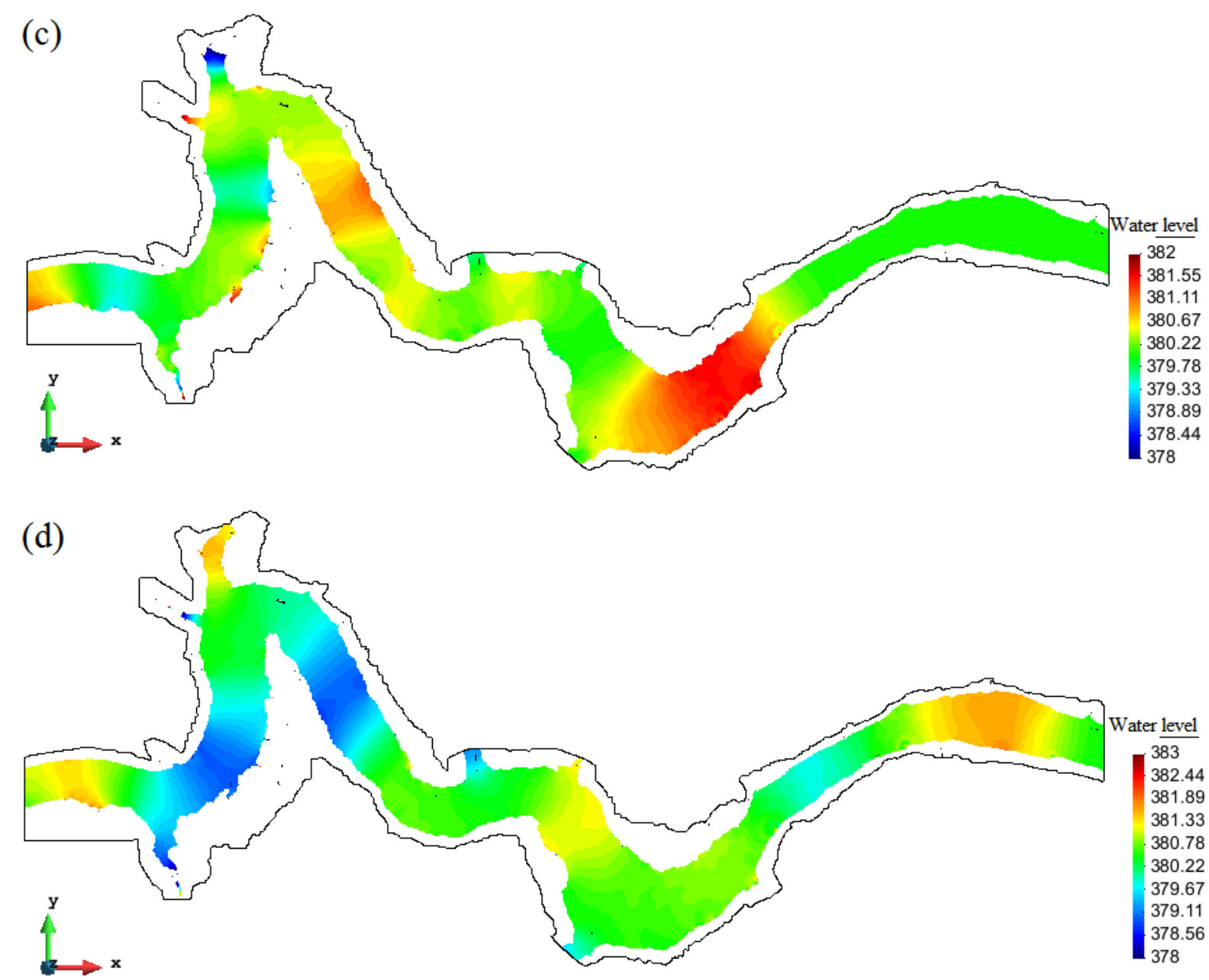

Fig. 12 Water level (m) at selected time in the whole domain (a) $t=30 \mathrm{~s}$; (b) $t=270 \mathrm{~s}$; (c) $t=500 \mathrm{~s}$; (d) $t=700 \mathrm{~s}$. Y points towards north.

The maximum incident primary wave amplitude in section 4 is $1.7 \mathrm{~m}$ and the free board is $4 \mathrm{~m}$. It is reasonable to believe that the Xiangjiaba Dam will not be overtopped by the wave caused by the Halaowo landslide with the selected parameters. Note that the secondary incident wave is likely to be smaller than the primary wave and the observed amplitude of 2.7 $\mathrm{m}$ is the superposition of the secondary incident and primary reflected wave from the dam.

Even though there is no experimental data about the Halaowo landslide to verify the outcome of the numerical results, the key point is to apply the proposed method to model the generation and propagation of the landslide and to predict the potential hazard. It is clear that this numerical method is able to simulate the whole process effectively.

\section{Conclusions}

A three-phases model has been proposed to simulate the generation and propagation of landslide-generated waves. Navier-Stokes equations coupled with a non-Newtonian 
viscoplastic fluid model for landslides were applied to model the strong interaction between three phases and the improved conservative level set method was expanded to $n$-phases to describe the interface characteristics accurately. All the equations were discretized with the characteristic-Garlerkin procedure. To verify the conservative feature for this method, the test case three-phases Zalesak slotted disk was used. Then, the simulation of the Lituya Bay waves was carried out and the current numerical results were compared with the existing published results. This method was finally used to model another practical project, namely, impulse waves generated by the Halaowo landslide near the Xiangjiaba Dam in the Jinsha River, including wave generation, propagation and run-up. It has been demonstrated that the proposed method is robust in modelling the characteristics of landslide-generated waves and it can be used to predict the potential hazard due to landslides.

\section{Acknowledgement}

This work is supported by the National Natural Science Foundation of China (No. 51279050), the Fundamental Research Funds for the Central Universities (No. 2016B05014), Fok Ying-Tong Education Foundation for Young Teachers in the Higher Education Institutions of China (No. 151073), Ministry of Water Resources non-profit specific industry appropriation (No. 201501036, No. 201501034 and No. 201501033), China Scholarship Council, and Qing Lan Project.

\section{References}

[1] Pelinovsky E, Poplavsky A. Simplified model of tsunami generation by submarine landslides. Phys Chem Earth, 1996;21(1):13-17.

[2] Noda E. Water waves generated by landslides. J Waterway Div, 1970;96(4):835-55.

[3] Lynett P, Liu PLF. A numerical study of the run-up generated by three-dimensional landslides. J Geophys Res-Oceans, 2005;110(C3).

[4] Panizzo A, De Girolamo P, Petaccia A. Forecasting impulse waves generated by subaerial landslides. J Geophys Res-Oceans, 2005;110(C12).

[5] Ataie-Ashtiani B, Nik-Khah A. Impulsive waves caused by subaerial landslides. Environ Fluid Mech, 2008;8(3):263-80.

[6] Di Risio M, De Girolamo P, Bellotti G, Panizzo A, Aristodemo F, Molfetta MG, Petrillo AF. Landslide-generated tsunamis runup at the coast of a conical island: New physical model experiments. J Geophys Res-Oceans, 2009;114(C1).

[7] Mohammed F, Fritz HM. Physical modeling of tsunamis generated by threedimensional deformable granular landslides. J Geophys Res-Oceans, 2012;117(C11).

[8] Heller V, Spinneken J. Improved landslide-tsunami prediction: Effects of block model parameters and slide model. J Geophys Res-Oceans, 2013;118(3):1489-507.

[9] Heller V, Spinneken J. On the effect of the water body geometry on landslidetsunamis: Physical insight from laboratory tests and 2D to 3D wave parameter transformation. Coastal Eng, 2015;104:113-34.

[10] Heller V, Hager WH, Minor H-E. Scale effects in subaerial landslide generated impulse waves. Exp Fluids, 2008;44:691. 
[11] Serrano-Pacheco A, Murillo J, García-Navarro P. A finite volume method for the simulation of the waves generated by landslides. J Hydrol, 2009;373(3-4):273-89.

[12] Shokina N, Aizinger V. On numerical modelling of impulse water waves generated by submarine landslides. Environ Earth Sci, 2015;74(11):7387-405.

[13] Mao J, Zhao L, Bai X, Guo B, Liu Z, Li T. A novel well-balanced scheme for modeling of dam break flow in drying-wetting areas. Comput Fluids, 2016;136:32430 .

[14] Ataie-Ashtiani B, Najafi Jilani A. A higher-order Boussinesq-type model with moving bottom boundary: applications to submarine landslide tsunami waves. Int J Numer Methods Fluids, 2007;53(6):1019-48.

[15] Ataie-Ashtiani B, Yavari-Ramshe S. Numerical simulation of wave generated by landslide incidents in dam reservoirs. Landslides, 2011;8(4):417-32.

[16] Huang B, Yin Y, Du C. Risk management study on impulse waves generated by Hongyanzi landslide in Three Gorges Reservoir of China on June 24, 2015. Landslides, 2016;13(3):603-16.

[17] Grilli ST, Watts P. Modeling of waves generated by a moving submerged body. Applications to underwater landslides. Eng Anal Boundary Elem, 1999;23(8):645-56.

[18] Grilli ST, Vogelmann S, Watts P. Development of a 3D numerical wave tank for modeling tsunami generation by underwater landslides. Eng Anal Boundary Elem, 2002;26(4):301-13.

[19] Abadie S, Morichon D, Grilli S, Glockner S. Numerical simulation of waves generated by landslides using a multiple-fluid Navier-Stokes model. Coastal Eng, 2010;57(9):779-94.

[20] Ma G, Shi F, Kirby JT. Shock-capturing non-hydrostatic model for fully dispersive surface wave processes. Ocean Model, 2012;43-44:22-35.

[21] Horrillo J, Wood A, Kim GB, Parambath A. A simplified 3-D Navier-Stokes numerical model for landslide-tsunami: Application to the Gulf of Mexico. J Geophys Res-Oceans, 2013;118(12):6934-50.

[22] López-Venegas AM, Horrillo J, Pampell-Manis A, Huérfano V, Mercado A. Advanced tsunami numerical simulations and energy considerations by use of 3D-2D coupled models: The October 11, 1918, Mona Passage Tsunami. Pure Appl Geophys, 2015;172(6):1679-98.

[23] Yim SC, Yuk D, Panizzo A, Di Risio M, Liu PLF. Numerical simulations of wave generation by a vertical plunger using RANS and SPH models. J Waterw Port C, 2008;134(3):143-59.

[24] Grilli ST, Guyenne P, Dias F. A fully non-linear model for three-dimensional overturning waves over an arbitrary bottom. Int $\mathrm{J}$ Numer Methods Fluids, 2001;35(7):829-67.

[25] Ward SN, Day S. Cumbre Vieja Volcano-Potential collapse and tsunami at La Palma, Canary Islands. Geophys Res Lett, 2001;28(17):3397-400.

[26] Haugen KB, Løvholt F, Harbitz CB. Fundamental mechanisms for tsunami generation by submarine mass flows in idealised geometries. Mar Pet Geol, 2005;22(1-2):209-17.

[27] Stéphan T. Grilli, Watts P. Tsunami generation by submarine mass failure. I: Modeling, experimental validation, and sensitivity analyses. J Waterw Port C, 2005;131(6).

[28] Yavari-Ramshe S, Ataie-Ashtiani B. Numerical modeling of subaerial and submarine landslide-generated tsunami waves-recent advances and future challenges. Landslides, 2016;13(6):1325-68.

[29] Yavari-Ramshe S, Ataie-Ashtiani B. A rigorous finite volume model to simulate subaerial and submarine landslide-generated waves. Landslides, 2017;14(1):203-21. 
[30] Quecedo M, Pastor M, Herreros MI. Numerical modelling of impulse wave generated by fast landslides. Int J Numer Methods Eng, 2004;59(12):1633-56.

[31] Zhao L, Mao J, Bai X, Liu X, Li T, Williams JJR. Finite element simulation of impulse wave generated by landslides using a three-phase model and the conservative level set method. Landslides, 2016;13(1):85-96.

[32] Monaghan JJ. Simulating free surface flows with SPH. J Comput Phys, 1994;110(2):399-406.

[33] Khanpour M, Zarrati AR, Kolahdoozan M, Shakibaeinia A, Jafarinik S. Numerical modeling of free surface flow in hydraulic structures using Smoothed Particle Hydrodynamics. Appl Math Model, 2016;40(23-24):9821-34.

[34] Farhadi A, Emdad H, Rad EG. Incompressible SPH simulation of landslide impulsegenerated water waves. Nat Hazards, 2016;82(3):1779-802.

[35] Heller V, Bruggemann M, Spinneken J, Rogers BD. Composite modelling of subaerial landslide-tsunamis in different water body geometries and novel insight into slide and wave kinematics. Coastal Eng, 2016;109:20-41.

[36] Liu PLF, Wu TR, Raichlen F, Synolakis CE, Borrero JC. Runup and rundown generated by three-dimensional sliding masses. J Fluid Mech, 2005;536:107-44.

[37] Montagna F, Bellotti G, Di Risio M. 3D numerical modeling of landslide-generated tsunamis around a conical island. Nat Hazards, 2011;58(1):591-608.

[38] James N, Boyaval S, Caboussat A, Picasso M. Numerical simulation of 3D free surface flows, with multiple incompressible immiscible phases. Applications to impulse waves. Int J Numer Methods Fluids, 2014;76(12):1004-24.

[39] Osher S, Sethian JA. Fronts propagating with curvature-dependent speed: Algorithms based on Hamilton-Jacobi formulations. J Comput Phys, 1988;79(1):12-49.

[40] Sussman M, Smereka P, Osher S. A level set approach for computing solutions to incompressible two-phase flow. J Comput Phys, 1994;114(1):146-59.

[41] Olsson E, Kreiss G. A conservative level set method for two phase flow. J Comput Phys, 2005;210(1):225-46.

[42] Olsson E, Kreiss G, Zahedi S. A conservative level set method for two phase flow II. J Comput Phys, 2007;225(1):785-807.

[43] Zhao L, Bai X, Li T, Williams JJR. Improved conservative level set method. Int J Numer Methods Fluids, 2014;75(8):575-90.

[44] Zhao L-h, Mao J, Liu X-q, Bai X, Williams JJR. Improved conservative level set method for free surface flow simulation. J Hydrodyn, 2014;26(2):316-25.

[45] Chen C-1, Ling C-H. Granular-flow rheology: role of shear-rate number in transition regime. J Eng Mech, 1996;122(5):469-80.

[46] Sussman M, Fatemi E, Smereka P, Osher S. An improved level set method for incompressible two-phase flows. Comput Fluids, 1998;27(5):663-80.

[47] Zienkiewicz OC, Taylor RL, Nithiarasu P, The finite element method for fluid dynamics (seventh edition), Oxford: Butterworth-Heinemann, 2014.

[48] Chorin AJ. A numerical method for solving incompressible viscous flow problems. J Comput Phys, 1997;135(2):118-25.

[49] Fritz HM, Hager WH, Minor H-E. Lituya Bay case: Rockslide impact and wave runup. Sci Tsunami Hazards, 2001;19:3-22.

[50] Miller DJ, Giant waves in Lituya Bay, Alaska, Report 354C, 1960.

[51] Fritz HM, Mohammed F, Yoo J. Lituya Bay landslide impact generated mega-tsunami 50th anniversary. Pure Appl Geophys, 2009;166(1):153-75.

[52] Weiss R, Fritz HM, Wünnemann K. Hybrid modeling of the mega-tsunami runup in Lituya Bay after half a century. Geophys Res Lett, 2009;36:1-6. 
[53] Rzadkiewicz SA, Mariotti C, Heinrich P. Numerical simulations of submarine landslides and their hydraulic effects. J Waterw Port C, 1997;123:149-57. 